\title{
Sparse Dose Painting Based on a Dual-Pass Kinetic-Oxygen Mapping of Dynamic PET Images
}

\author{
Kuangyu Shi ${ }^{1}$, Sabrina T. Astner ${ }^{1}$, Liang Sun ${ }^{2}$, Nassir Navab $^{3}$, Fridtjof Nüsslin ${ }^{1}$, \\ Peter Vaupel ${ }^{1}$, and Jan J. Wilkens ${ }^{1}$ \\ ${ }^{1}$ Radiation Oncology, Technische Universität München, Munich, Germany \\ ${ }^{2}$ Computer Science and Engineering, Arizona State University, Tempe, USA \\ ${ }^{3}$ Computer Aided Medical Procedures, Technische Universität München, Munich, Germany \\ shi@lrz.tum.de
}

\begin{abstract}
Development of molecular imaging such as positron-emission tomography (PET) offers an opportunity to optimize radiotherapy treatment planning by conforming the dose distribution to physiological details within tumors, so called dose painting. Quantification of the acquired images and an efficient and practical dose prescription remain two key questions in this field. This paper proposes a novel framework to optimize the dose prescription based on dual-pass modeling of dynamic $\left[{ }^{18} \mathrm{~F}\right] \mathrm{FMISO}$ PET images. An optimization algorithm for sparse dose painting (SDP) is developed by minimizing a linear combination of two terms corresponding to the efficiency and total variation of the dose distribution with the constraint of a constant mean dose. Dose efficiency is defined using the linear-quadratic model. The radiosensitivity given by the oxygen tension is estimated using a dual-pass kinetic-oxygen mapping strategy. This is achieved by integrating a realistic $\left[{ }^{18} \mathrm{~F}\right] \mathrm{FMISO}$ PET imaging simulation model, which can simulate the distribution of oxygen and tracer under the same tumor microenvironment setting. The algorithm was compared with a typical dose painting by number (DPBN) method in one data set of a patient with head and neck cancer.
\end{abstract}

Keywords: Dose Painting, Sparse Optimization, Radiation Therapy, Computational Simulation, Positron Emission Tomography.

\section{Introduction}

Medical imaging plays an important role in radiotherapy including treatment planning [1|2], delivery [3] and monitoring. Recent developments have focused on the incorporation of image derived physiological information into treatment planning [12]. Molecular imaging modalities, such as positron-emission tomography (PET), can be applied to improve the therapy outcome by conforming the dose distribution to the heterogeneous tumor microenvironment, which is called dose painting [4]. Inverse planning and intensity-modulated radiotherapy (IMRT) with dynamic multileaf collimators are applied to shape the delivery to match the dose prescription [5]. To prescribe a proper and efficient dose distribution based on the acquired molecular images is one key factor for successful dose painting.

In practice, two strategies have been applied to paint dose values within a tumor: (1) boost escalation, which outlines the resistant regions and escalates uniform doses

G. Fichtinger, A. Martel, and T. Peters (Eds.): MICCAI 2011, Part I, LNCS 6891, pp. 484 491, 2011.

(c) Springer-Verlag Berlin Heidelberg 2011 
correspondingly; (2) dose painting by numbers (DPBN), which prescribes the dose according to the radiosensitivity difference of each image voxel [67]. Concerning the highly heterogeneous tumor microenvironment [8], DPBN has advantages in conformance to biological details. However, it is often hardly feasible in practice due to the exploded complexities in planning and delivery procedure, which not only increases the burden to patients and machines, but also reduces the freedom of normal tissue sparing. To prescribe a practical dose distribution while keeping the efficiency remains challenging in dose painting. Sparsity regularization is one booming technology to explore the information usage while reducing the data complexity, which is now popular in the application of image reconstruction [9] and data analysis [10]. For the application in dose painting, proper regularization terms need to be integrated during the optimization of dose efficiency.

Radiation response is strongly influenced by tumor oxygenation, which is usually assessed using $\left[{ }^{18} \mathrm{~F}\right]$ FMISO PET images. Kinetic modeling is often used to assess tracer pharmacokinetic parameters [11] and has been reported to be of advantage compared to direct evaluation of static $\left[{ }^{18} \mathrm{~F}\right] \mathrm{FMISO}$ PET images [11]. However, it is usually not possible to quantitatively relate the estimated kinetic parameters directly to the underlying oxygen tensions due to the complex tracer transport and metabolism process in the heterogeneous tumor microenvironment. On the other hand, a recently developed simulation model based on reaction-diffusion equations by Kelly et al. [12] is able to simulate the distributions of tracer and oxygen under the same tumor microenvironment, thus it is possible to explore the relation between $\left[{ }^{18} \mathrm{~F}\right] \mathrm{FMISO}$ imaging and oxygen tension. An extension of the Kelly model called flow-limited oxygen-dependent (FLOD) model has been validated with preclinical PET data [13].

This paper proposes a framework to optimize the dose distribution based on quantitative analysis of $\left[{ }^{18} \mathrm{~F}\right] \mathrm{FMISO}$ PET image series. We developed a sparse dose painting (SDP) algorithm by optimizing a linear combination of two terms corresponding to the efficiency and total variation of the dose distribution under the constraint of constant mean dose, which comprises the therapy outcome and the treatment complexity. The radiosensitivity parameter, oxygen tension, is estimated using one dual-pass kineticoxygen mapping strategy, which is achieved by simulating the transport and metabolism of oxygen and tracer under the same condition and setting up a kinetic-oxygen mapping curve based on the simulation results of various tumor microenvironments.

\section{Sparse Dose Painting}

One aim in dose painting is to compute a proper dose distribution $\mathbf{D}$ in a tumor domain $\Omega$ based on the estimated oxygen tensions from $\left[{ }^{18} \mathrm{~F}\right] \mathrm{FMISO}$ PET images. Given a certain prescribed mean dose $D_{\text {mean }}$, treatment planning attempts to optimize the dose $D(\mathbf{u})$ of each image voxel $\mathbf{u}, \mathbf{u} \in \Omega$ and achieve a maximum treatment efficiency, i.e. a minimum fraction of surviving cells after irradiation (surviving fraction $S F$ ). However, IMRT is usually done with a limited number of beams and restricted range of intensity modulation leading to a sparse dose prescription. An additional term $\|\mathbf{D}\|_{T V}=\sum_{i, j, k \in \Omega}\left(\left(\nabla_{1} D_{i j k}\right)^{2}+\left(\nabla_{2} D_{i j k}\right)^{2}+\left(\nabla_{3} D_{i j k}\right)^{2}\right)$ is introduced to regularize the total variation (TV) and to reduce the complexity of the dose distribution. Here $\nabla_{1}$, 
$\nabla_{2}$ and $\nabla_{3}$ denote the forward finite difference operators on the three coordinates, respectively. The optimization metric can be described in the following mathematic form,

$$
\arg \min _{\mathbf{D}}\left\{\log \left(\|S F(\mathbf{D})\|_{1}\right)+\lambda \frac{\|\mathbf{D}\|_{T V}}{N_{\text {voxel }}}\right\} \text { subject to mean }(\mathbf{D})=D_{\text {mean }}
$$

where $\lambda$ is the regularization parameter, which is patient-specific concerning the patient tolerance, tumor geometry and the topology with the surrounding normal tissue.

The surviving fraction $S F(\mathbf{u})$ of each voxel is determined by the linear-quadratic model, which is an empirical formula for the predication of radiation response [14]:

$$
\begin{aligned}
S F(\mathbf{u}) & =e^{-\alpha(\bar{p}(\mathbf{u})) D(\mathbf{u})-\beta(\bar{p}(\mathbf{u})) D(\mathbf{u})^{2}} \\
\alpha(\bar{p}(\mathbf{u})) & =\alpha_{0} \frac{m \bar{p}(\mathbf{u})+K}{\bar{p}(\mathbf{u})+K} \\
\beta(\bar{p}(\mathbf{u})) & =\beta_{0}\left(\frac{m \bar{p}(\mathbf{u})+K}{\bar{p}(\mathbf{u})+K}\right)^{2}
\end{aligned}
$$

$\alpha$ and $\beta$ are the radiation sensitivity coefficients, which are determined by the mean oxygen tension $\bar{p}$ of imaging voxels u. $\alpha_{0}, \beta_{0}, m$ and $K$ are constants. Eqn 1 is solved using a conjugate gradient method [15] with the initialization of DPBN applied to the results of kinetic modeling.

\section{Dual-Pass Kinetic-Oxygen Mapping}

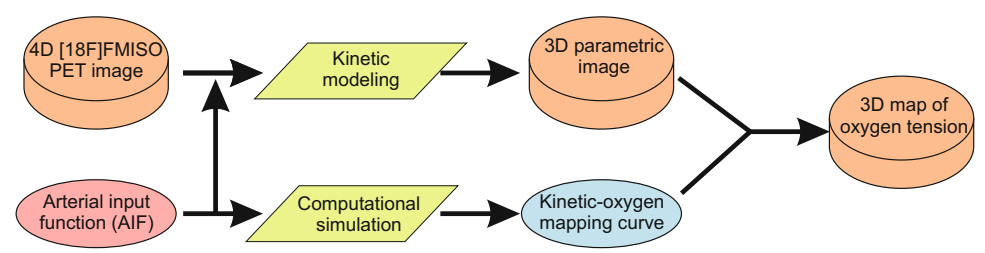

Fig. 1. The procedure of dual-pass kinetic-oxygen mapping.

One prerequisite to solve Eqn.1 is to quantitatively estimate the radiosensitivity parameter, oxygen tension, from the acquired $\left[{ }^{18} \mathrm{~F}\right] \mathrm{FMISO}$ PET images. Kinetic modeling is often used for quantitative analysis of PET images [11|16]. However, the estimated parameters are still not directly related to oxygen tension. On the other hand, computational simulation of $\left[{ }^{18} \mathrm{~F}\right] \mathrm{FMISO}$ PET imaging has advantages in revealing the correlation between $\left[{ }^{18} \mathrm{~F}\right]$ FMISO kinetics and oxygen tension by computing their distribution under the same tumor microenvironment [12]. We developed a strategy to combine both the forward and backward modeling and to quantify the oxygen tension from dynamic PET images. The procedure is sketched in Fig. 1, A kinetic-oxygen mapping curve is derived by fitting the computational simulation results using the following formula,

$$
k_{3}=\frac{a}{\bar{p}+b}+c
$$


where $k_{3}$ is the estimated kinetic parameter using a typical irreversible two-compartment model [16] and $\bar{p}$ is the mean oxygen tension of a voxel $\mathbf{u}$. Constants of $a, b, c$ reflect the integral of the heterogeneous tracer binding procedure. They vary for different patients or animals due to different metabolic properties. The mapping curve of Eqn. 3 is then applied to transform the parametric image to the oxygen tension image.

\section{Reaction-Diffusion Simulation Model}

To identify the constants of Eqn. 3, we use an extension of the Kelly model [12] called flow-limited oxygen-dependent (FLOD) model to simulate the relation between the oxygen consumption and the $\left[{ }^{18} \mathrm{~F}\right]$ FMISO binding inside an imaging voxel in heterogeneous tumor microenvironments [13]. Tumor microenvironments are modeled as 2D grids (grid size $10 \mu \mathrm{m}$ ) spreading with heterogeneously distributed vessels. The transport and metabolism of oxygen and $\left[{ }^{18} \mathrm{~F}\right] \mathrm{FMISO}$ molecules are modeled as a diffusionreaction procedure with an assumption of flow-limited extraction. Given the physical and metabolic properties of oxygen and $\left[{ }^{18} \mathrm{~F}\right] \mathrm{FMISO}$ molecules measured from in vitro experiments, the distribution of oxygen and $\left[{ }^{18} \mathrm{~F}\right] \mathrm{FMISO}$ in tumor areas can be computed by solving the following reaction-diffusion equations:

$$
\begin{aligned}
\frac{\partial \operatorname{Tr}_{f r e e}(\mathbf{x}, t)}{\partial t} & =D_{T r} \nabla^{2} \operatorname{Tr}_{f r e e}(\mathbf{x}, t)-k_{\text {binding }}(\mathbf{x}) \operatorname{Tr}_{f r e e}(\mathbf{x}, t) \\
\frac{\partial \operatorname{Tr}_{\text {bound }}(\mathbf{x}, t)}{\partial t} & =k_{\text {binding }}(\mathbf{x}) \operatorname{Tr}_{f r e e}(\mathbf{x}, t) \\
k_{\text {binding }}(\mathbf{x}) & =\frac{k_{a}}{p(\mathbf{x})+k_{b}} \\
D_{\mathrm{O}_{2}} \nabla^{2} p(\mathbf{x}) & =-q_{\max } \frac{p(\mathbf{x})}{p(\mathbf{x})+k_{p}}
\end{aligned}
$$

where $\operatorname{Tr}_{\text {free }}(\mathbf{x}, t)$ describes the concentration of the free ligand for a spatial location $\mathbf{x}$ at time $t$, and $\operatorname{Tr}_{\text {bound }}(\mathbf{x}, t)$ is the concentration of the bound ligand. $k_{\text {binding }}(\mathbf{x})$ is the binding rate of the tracer, which relates to the cellular oxygen tension $p(\mathbf{x})$. $D_{T r}$ and $D_{\mathrm{O}_{2}}$ are diffusion coefficients of $\left[{ }^{18} \mathrm{~F}\right] \mathrm{FMISO}$ and oxygen. $q_{\max }$ and $k_{p}$ are oxygen metabolic constants. $k_{a}$ and $k_{b}$ are constants determined by $\left[{ }^{18} \mathrm{~F}\right] \mathrm{FMISO}$ pharmacodynamics. Note that these cellular parameters are different from the macroscopic parameters $a, b, c$ in Eqn. 3 . Further, the microscopic binding parameter $k_{\text {binding }}$ is different from the macroscopic estimation parameter $k_{3}$ using kinetic modeling.

\section{Experiments and Results}

We tested our algorithm on one data set of a patient with head and neck cancer, which was scanned with CT and a dynamic $\left[{ }^{18} \mathrm{~F}\right]$ FMISO PET. The image series consist of 30 continuous frames of the first $15 \mathrm{~min}$ post injection (p. i.) of $\left[{ }^{18} \mathrm{~F}\right] \mathrm{FMISO}$ and an additional frame at 2 hours p. i.. Two blood samples were acquired after the PET scan. The arterial input function (AIF) is derived using an image based method by selection of a carotid artery in the $\left[{ }^{18} \mathrm{~F}\right] \mathrm{FMISO}$ PET images followed by a correction of the partial 
volume effect. The measured blood activity is applied to correct the spill over effect by replacing the tail part of the image derived AIF. A three exponential model is applied to smooth the combined AIF [17]. The PET images were registered to the CT images using a mutual information algorithm. The tumor regions (primary tumor and affected lymph nodes) were outlined manually by an experienced radiation oncologist. Fig. 2 a shows a slice of the fused CT and PET images.

Fig. 2 2 shows the results of the analysis of the dynamic $\left[{ }^{18}\right.$ F]FMISO PET images using the dual-pass kinetic-oxygen mapping method. Fig. 2b depicts the estimated $k_{3}$ image of the slice in Fig. 2h. Around 400 PET imaging voxels (voxel size: $4 \times 4 \mathrm{~mm}^{2}$ ) were simulated based on the extracted AIF. The $k_{3}$ values of the simulated TACs and the corresponding mean oxygen tensions are plotted in Fig. 2. F. The kinetic-oxygen mapping curve is generated by fitting the simulation results using Eqn. 3. A slice of the map of the oxygen tension after kinetic-oxygen mapping is illustrated in Fig. 2 $\mathrm{d}$.

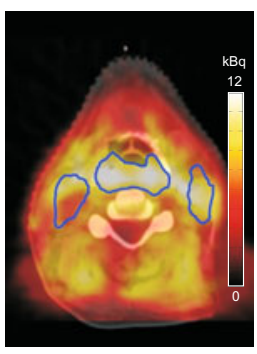

(a)

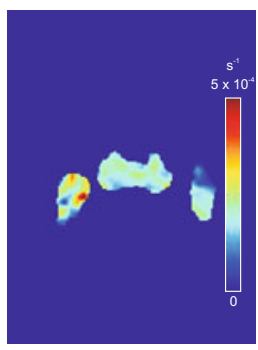

(b)

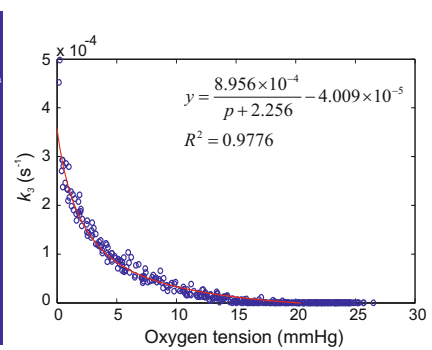

(c)

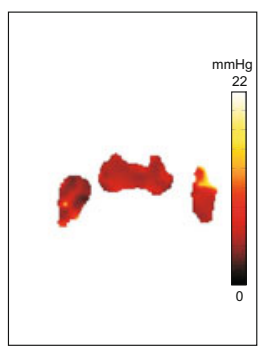

(d)

Fig. 2. Results of the dual-pass kinetic-oxygen mapping: (a) slice of the fusion of the CT image and the $\left[{ }^{18} \mathrm{~F}\right]$ FMISO PET image 2 hours p.i.; (b) parametric image of $k_{3}$ using pixel-wise irreversible two-compartment model within the tumor regions for slice a; (c) plot of the values of the kinetic parameters $k_{3}$ and the mean oxygen tensions of 400 simulated voxels and the resulting kinetic-oxygen mapping curve fitted using Eqn. 3. (d) reconstructed oxygen tension map within the tumor regions for slice a.

SDP with different $\lambda$ values was tested and compared with direct DPBN, which uses a linear transformation of static $\left[{ }^{18} \mathrm{~F}\right] \mathrm{FMISO}$ images to the dose prescription [6]. The prescribed dose distributions using SDP and DPBN were compared for the prescribed mean dose of $D_{\text {mean }}=2.3 \mathrm{~Gy}$ for a single fraction within the tumor regions here. One example slice of different resulting dose prescriptions is displayed in Fig $3 \mathrm{~h}$-e. Even without sparsity regularization, dose painting based on the dual-pass kinetic-oxygen quantification (Fig. 3a) generates significantly different results compared to DPBN (Fig. 3 ). It confirms that a direct evaluation of PET image may not be sufficient to assess the underlying oxygen tension [11].

Fig. 3 $\mathrm{k}$-d show the comparison of increasing $\lambda$ values, which will reduce the complexity of dose prescription correspondingly. The regulated dose prescription has several advantages: (1) it will diminish the burden of inverse planning; (2) it will reduce

\footnotetext{
${ }^{1}$ The DPBN presented in [7] is not considered in this paper. The comparison of different tumor control probability models is not feasible in this framework.
} 


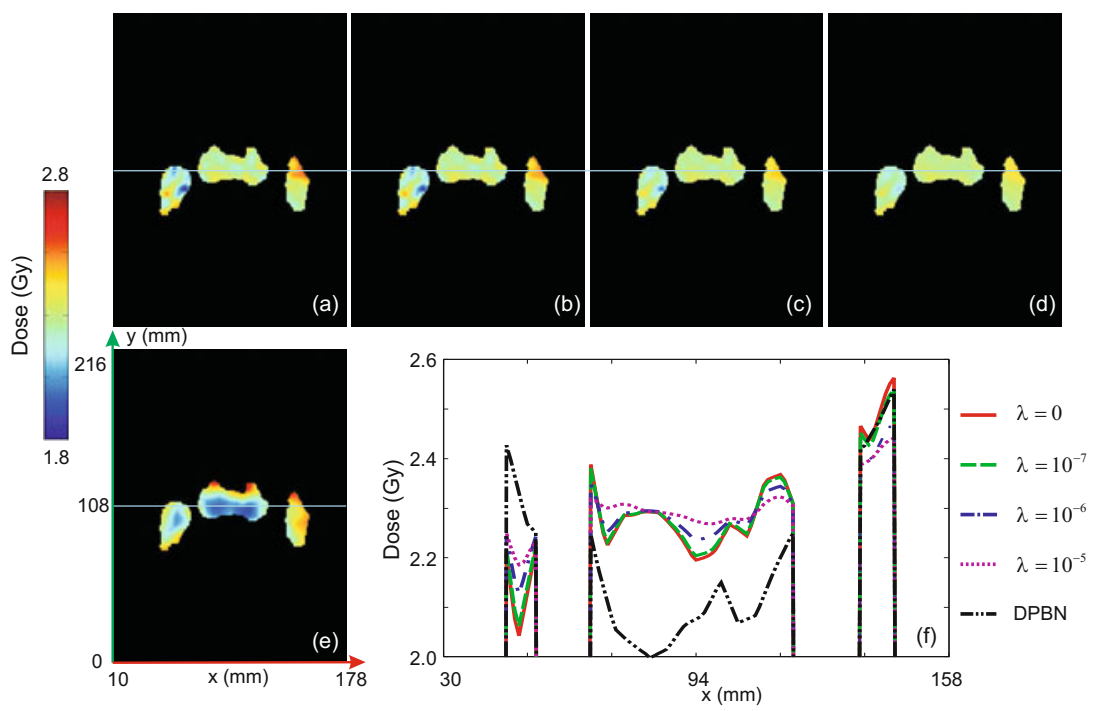

Fig. 3. Comparison of SDP with different $\lambda$ and DPBN for the patient case: (a-d) One slice of SDP dose prescription, $\lambda=0,10^{-7}, 10^{-6}, 10^{-5}$, respectively; (e) One slice of DPBN dose prescription; (f) A plot of the dose values along the blue lines in the middle of the selected slice shown in a-e.

Table 1. Comparison of the resulting average surviving fraction of different methods

\begin{tabular}{c|l|l|l|l}
\hline \multirow{2}{*}{ DPBN } & \multicolumn{4}{|c}{ SDP } \\
\cline { 2 - 5 } & $\lambda=0$ & $\lambda=10^{-7}$ & $\lambda=10^{-6}$ & $\lambda=10^{-5}$ \\
\hline $2.99 \times 10^{-4}$ & $2.61 \times 10^{-6}$ & $4.69 \times 10^{-6}$ & $1.17 \times 10^{-4}$ & $1.22 \times 10^{-4}$ \\
\hline
\end{tabular}

$\lambda$ : regularization parameter; SDP: sparse dose painting; DPBN: dose painting by number.

the number of beams and the complexity of fluence modulation leading to a reduced treatment time; (3) it will improve the quality of normal tissues sparing; besides treating the tumor, radiotherapy needs to avoid too much side effects in normal tissues. For a constant total dose, SDP reduces extremely high dose prescriptions in the tumor (shown in Fig. 3). Thus it is also possible to reduce the probability of hot spots in the normal tissue. Overall, the sparsity regularization will increase the feasibility of dose painting. The corresponding dose efficiency after 30 fractions is listed in Tab1 SDP has a better dose efficiency compared to DPBN due to the improved quantification of the oxygen tensions. Note, due to the large values of the TV norm, the $\lambda$ values are quite small here to ensure a proper regularization. A suitable $\lambda$ value is determined by various factors, such as the volume and geometry of the tumor and the topology with the surrounding normal tissues. It can be adapted in real applications and will be optimized based on statistical analysis of further studies. 


\section{Conclusion}

This paper develops a novel framework to optimize the dose prescription in dose painting based on quantitative analysis of $\left[{ }^{18} \mathrm{~F}\right]$ FMISO PET images. In particular, we made the following two contributions: (1) By incorporating a realistic PET imaging simulation model, this paper sets up a dual-pass kinetic-oxygen mapping to transform the estimated kinetic parameters to physiological parameters of the tumor microenvironment. (2) A sparse dose painting algorithm is developed to optimize the dose efficiency as well as the dose modulation. This is achieved by adding a sparsity regularization term during the optimization of dose efficiency with the constraint of constant mean dose.

Our method is limited by the indirect quantification by integrating computational simulation into tracer kinetic modeling, which might induce bias and errors. (1) Although the FLOD simulation model is in good agreement with preclinical experimental data, for clinical applications it is necessary to further adjust and validate the model settings. (2) The two-pass quantification procedure may accumulate and amplify errors in some situations. Direct matching of reaction-diffusion simulation to imaging data is desired [2]. However, the tracer uptake procedure is determined by both the spatial location and the perfusion of vessels spreading into tissues discretely and randomly, which is generally not differentiable. Nevertheless, our method offers the potential to improve the treatment outcome of dose painting. Future work will focus on the further optimization of dose prescription in the clinical target volume by integrating tumor growth parameters [2] in a tumor control probability model and on the testing and integration of the algorithm in real therapy planning systems.

Acknowledgements. Supported by BMBF MobiTUM project (01EZ0826) and DFG Cluster of Excellence: Munich-Centre for Advanced Photonics.

\section{References}

1. Konukoğlu, E., Clatz, O., Bondiau, P.-Y., Delingette, H., Ayache, N.: Extrapolating tumor invasion margins for physiologically determined radiotherapy regions. In: Larsen, R., Nielsen, M., Sporring, J. (eds.) MICCAI 2006. LNCS, vol. 4190, pp. 338-346. Springer, Heidelberg (2006)

2. Konukoglu, E., Clatz, O., Menze, B.H., Stieltjes, B., Weber, M.A., Mandonnet, E., Delingette, H., Ayache, N.: Image Guided Personalization of Reaction-Diffusion Type Tumor Growth Models Using Modified Anisotropic Eikonal Equations. IEEE Trans. Med. Imaging 29, 77-95 (2010)

3. Lu, C., Chelikani, S., Chen, Z., Papademetris, X., Staib, L.H., Duncan, J.S.: Integrated segmentation and nonrigid registration for application in prostate image-guided radiotherapy. In: Jiang, T., Navab, N., Pluim, J.P.W., Viergever, M.A. (eds.) MICCAI 2010. LNCS, vol. 6361, pp. 53-60. Springer, Heidelberg (2010)

4. Ling, C., Humm, J., Larson, S., Amols, H., Fuks, Z., Leibel, S., Koutcher, J.: Towards Multidimensional Radiotherapy (MD-CRT): Biological Imaging and Biological Conformality. Int. J. Radiat. Oncol. Biol. Phys. 47, 551-560 (2000)

5. Simari, P.D., Wu, B., Jacques, R., King, A., McNutt, T., Taylor, R., Kazhdan, M.M.: A statistical approach for achievable dose querying in IMRT planning. In: Jiang, T., Navab, N., Pluim, J.P.W., Viergever, M.A. (eds.) MICCAI 2010. LNCS, vol. 6363, pp. 521-528. Springer, Heidelberg (2010) 
6. Alber, M., Paulsen, F., Eschmann, S.M., Machulla, H.J.: On Biologically Conformal Boost Dose Optimization. Phys. Med. Biol. 35, N31-N35 (2003)

7. Thorwarth, D., Eschmann, S., Paulsen, F., Alber, M.: Hypoxia Dose Painting by Numbers: a Planning Study. Int. J. Radiat. Oncol. Biol. Phys. 68, 291-300 (2007)

8. Vaupel, P.: Tumor Microenvironmental Physiology and its Implications for Radiation Oncology. Semin. Radiat. Oncol. 14, 198-206 (2004)

9. Huang, J., Zhang, S., Metaxas, D.N.: Efficient MR image reconstruction for compressed MR imaging. In: Jiang, T., Navab, N., Pluim, J.P.W., Viergever, M.A. (eds.) MICCAI 2010. LNCS, vol. 6361, pp. 135-142. Springer, Heidelberg (2010)

10. Avants, B.B., Cook, P.A., McMillan, C., Grossman, M., Tustison, N.J., Zheng, Y., Gee, J.C.: Sparse unbiased analysis of anatomical variance in longitudinal imaging. In: Jiang, T., Navab, N., Pluim, J.P.W., Viergever, M.A. (eds.) MICCAI 2010. LNCS, vol. 6361, pp. 324-331. Springer, Heidelberg (2010)

11. Thorwarth, D., Eschmann, S., Paulsen, F., Alber, M.: A Kinetic Model for Dynamic ${ }^{18}$ FFmiso PET Data to Analyse Tumour Hypoxia. Phys. Med. Biol. 50, 2209-2224 (2005)

12. Kelly, C., Brady, M.: A Model to Simulate Tumour Oxygenation and Dynamic $\left[{ }^{18} \mathrm{~F}\right]-\mathrm{Fmiso}$ PET Data. Phys. Med. Biol. 51, 5859-5873 (2006)

13. Shi, K., Bayer, C., Maftei, C.A., Gaertner, F.C., Astner, S.T., Wilkens, J.J., Nüsslin, F., Vaupel, P., Ziegler, S.I.: A Flow-limited Oxygen-dependent Diffusion Model with Heterogeneous Perfusion for Quantitative Analysis of Dynamic $\left[{ }^{18} \mathrm{~F}\right]$ Fmiso PET Uptake in Tumors (submitted)

14. Wenzl, T., Wilkens, J.J.: Modelling of the Oxygen Enhancement Ratio for Ion Beam Radiation Therapy. Phys. Med. Biol. 56, 3251-3268 (2011)

15. Nocedal, J., Wright, S.J.: Numerical Optimization, 2nd edn. Springer, Heidelberg (2000)

16. Shi, K., Souvatzoglou, M., Astner, S.T., Vaupel, P., Nüsslin, F., Wilkens, J.J., Ziegler, S.I.: Quantitative Assessment of Hypoxia Kinetic Models by a Cross-Study of Dynamic ${ }^{18} \mathrm{~F}-$ FAZA and ${ }^{18} \mathrm{O}-\mathrm{H}_{2} \mathrm{O}$ in Patients with Head and Neck Tumors. J. Nucl. Med. 51, 1386-1394 (2010)

17. Feng, D., Huang, S., Wang, X.: Models for Computer Simulation Studies of Input Functions for Tracer Kinetic Modeling with Positron Emission Tomography. Int. J. Biomed. Comput. 32, 95-110 (1993) 